\title{
Stability of a Quartic Functional Equation in Restricted Domains
}

\author{
Jaeyoung Chung and Yu-Min Ju \\ Department of Mathematics, Kunsan National University, Kunsan 573-701, Republic of Korea \\ Correspondence should be addressed to Jaeyoung Chung; jychung@kunsan.ac.kr \\ Received 24 March 2016; Revised 11 May 2016; Accepted 16 May 2016 \\ Academic Editor: Zbigniew Leśniak
}

Copyright (C) 2016 J. Chung and Y.-M. Ju. This is an open access article distributed under the Creative Commons Attribution License, which permits unrestricted use, distribution, and reproduction in any medium, provided the original work is properly cited.

Let $X$ be a real normed space and $Y$ a Banach space and $f: X \rightarrow Y$. We prove the Ulam-Hyers stability theorem for the quartic functional equation $f(2 x+y)+f(2 x-y)-4 f(x+y)-4 f(x-y)-24 f(x)+6 f(y)=0$ in restricted domains. As a consequence we consider a measure zero stability problem of the above inequality when $f: \mathbb{R} \rightarrow Y$.

\section{Introduction}

Let $\mathbb{R}, X$, and $Y$ be the set of real numbers, a real normed space, and a real Banach space, respectively. A mapping $f$ : $X \rightarrow Y$ is called a quartic mapping if $f$ satisfies the functional equation

$$
\begin{gathered}
f(2 x+y)+f(2 x-y)-4 f(x+y)-4 f(x-y) \\
-24 f(x)+6 f(y)=0
\end{gathered}
$$

for all $x, y \in X$. It is known in [1, Theorem 2.1] that the general solutions $f$ of (1) are given by $f(x)=B(x, x, x, x)$ for all $x \in$ $X$, where $B: X \times X \times X \times X \rightarrow Y$ is a symmetric function which is additive for each variable when the other three variables are fixed. The following is a modified version of [1, Theorem 3.1]. We refer the reader to $[2,3]$ for the stability of generalized quartic mappings.

Theorem 1. Let $\epsilon \geq 0$ be fixed. Suppose that $f: X \rightarrow Y$ satisfies the cubic functional inequality

$$
\begin{aligned}
& \| f(2 x+y)+f(2 x-y)-4 f(x+y)-4 f(x-y) \\
& \quad-24 f(x)+6 f(y) \| \leq \epsilon
\end{aligned}
$$

for all $x, y \in X$. Then there exists a unique quartic mapping $T: X \rightarrow Y$ such that

$$
\|f(x)-T(x)\| \leq \frac{\epsilon}{24}
$$

for all $x \in X$.
It is a very natural subject to study functional equations or inequalities satisfied on restricted domains or satisfied under restricted conditions [4-19]. Among the results, Jung [14] and Rassias [17] proved the Ulam-Hyers stability of the quadratic functional equations in a restricted domain. As a refined version of the results in $[14,17]$ we state the result in $[20]$.

Theorem 2. Let $d>0$. Suppose that $f: X \rightarrow Y$ satisfies the inequality

$$
\|f(x+y)+f(x-y)-2 f(x)-2 f(y)\| \leq \delta
$$

for all $x, y \in D:=\{(x, y) \in X \times X:\|x\|+\|y\| \geq d\}$. Then there exists a unique mapping $q: X \rightarrow Y$ satisfying

$$
f(x+y)+f(x-y)=2 f(x)+2 f(y)
$$

for all $x, y \in X$ such that

$$
\|f(x)-q(x)\| \leq \frac{3}{2} \epsilon
$$

for all $x \in X$.

Also, Chung-Ju-Rassias [21] proved the following UlamHyers stability of cubic functional equation in restricted domains. 
Theorem 3. Let $d>0$. Suppose that $f: X \rightarrow Y$ satisfies the inequality

$$
\begin{aligned}
& \| f(2 x+y)+f(2 x-y)-2 f(x+y)-2 f(x-y) \\
& \quad-12 f(x) \| \leq \epsilon
\end{aligned}
$$

for all $x, y \in D:=\{(x, y) \in X \times X:\|x\|+\|y\| \geq d\}$. Then there exists a unique mapping $c: X \rightarrow Y$ satisfying

$$
\begin{gathered}
c(2 x+y)+c(2 x-y)-2 c(x+y)-2 c(x-y) \\
-12 c(x)=0
\end{gathered}
$$

for all $x, y \in X$ such that

$$
\|f(x)-c(x)-48 f(0)\| \leq \frac{79}{14} \epsilon
$$

for all $x \in X$.

In this paper, we consider the Ulam-Hyers stability of quartic functional equation (1) in some restricted domains $\Omega \subset X \times X$. First, imposing condition (C) on $\Omega$ (see Section 2) we prove that if $f: X \rightarrow Y$ satisfies inequality (2) for all $(x, y) \in \Omega$, then there exists a unique quartic mapping $T: X \rightarrow Y$ such that

$$
\|f(x)-T(x)+231 f(0)\| \leq \frac{509}{36} \epsilon
$$

for all $x \in X$. Since $\Omega=\{(x, y) \in X \times X:\|x\|+\|y\| \geq d\}$ satisfies condition $(\mathrm{C})$, we obtain the parallel result for quartic functional equation as Theorem 2 for a quadratic functional equation and Theorem 3 for a cubic functional equation.

Secondly, constructing a subset $\Gamma \subset \mathbb{R}^{2}$ of measure 0 satisfying condition $(C)$ we consider a measure zero stability problem of quartic functional equation (1) for all $x, y \in \Gamma$, where $f: \mathbb{R} \rightarrow Y$ and $\Gamma \subset\left\{(x, y) \in \mathbb{R}^{2}:|x|+|y| \geq d\right\}$ has 2-dimensional Lebesgue measure 0 .

As an application we consider an asymptotic behavior of $f: \mathbb{R} \rightarrow Y$ satisfying the weak condition

$$
\begin{aligned}
& \| f(2 x+y)+f(2 x-y)-4 f(x+y)-4 f(x-y) \\
& \quad-24 f(x)+6 f(y) \| \longrightarrow 0
\end{aligned}
$$

as $|x|+|y| \rightarrow \infty$ only for $(x, y) \in \Gamma$, where $\Gamma$ has 2dimensional Lebesgue measure 0 .

\section{Stability of the Quartic Functional Equation in Restricted Domain}

Throughout this section we assume that $\Omega \subset X \times X$ satisfies the following condition:

\section{For given $x, y \in X$ there exists $t \in X$ such that}

(C) $P_{x, y, t}:=\{(x-t, y+2 t),(x+t, y+2 t),(x-t,-y+2 t)$, $(x+t,-y+2 t),(x, y+4 t),(x,-y+4 t),(-t, x+y+2 t)$, $(t, x-y-2 t),(-t, x-y+2 t),(t, x+y-2 t),(-t, x+y+t)$, $(t, x-y-t),(-t, x-y+t),(t, x+y-t),(t, x+y)$,

$$
\begin{aligned}
& (-t, x-y),(t, x-y),(-t, x+y),(x+t, y),(x-t, y) \\
& (x, y+2 t),(x,-y+2 t),(0, x+y+4 t),(0, x+y-4 t), \\
& (0, x-y+4 t),(0, x-y-4 t),(0, x+y+3 t),(0, x+y-3 t) \\
& (0, x-y+3 t),(0, x-y-3 t),(0, x+y+2 t),(0, x+y-2 t) \\
& (0, x-y+2 t),(0, x-y-2 t),(0, x+y-t),(0, x+y+t), \\
& (0, x-y-t),(0, x-y+t),(2 t, y),(t, 0),(0, t)\} \subset \Omega .
\end{aligned}
$$

Theorem 4. Let $\epsilon \geq 0$ be fixed. Suppose that $f: X \rightarrow Y$ satisfies inequality (2) for all $(x, y) \in \Omega$. Then there exists a unique quartic mapping $T: X \rightarrow Y$ satisfying (10) for all $x \in X$.

Proof. Let

$$
\begin{aligned}
F(x, y)= & f(2 x+y)+f(2 x-y)-4 f(x+y) \\
& -4 f(x-y)-24 f(x)+6 f(y)
\end{aligned}
$$

for all $x, y \in X$. Then

$$
\begin{aligned}
& F(x-t, y+2 t)=f(2 x+y)+f(2 x-y-4 t) \\
& -4 f(x+y+t) \\
& -4 f(x-y-3 t) \\
& -24 f(x-t)+6 f(y+2 t), \\
& F(x+t, y+2 t)=f(2 x+y+4 t)+f(2 x-y) \\
& -4 f(x+y+3 t) \\
& -4 f(x-y-t) \\
& -24 f(x+t)+6 f(y+2 t), \\
& F(x-t,-y+2 t)=f(2 x-y)+f(2 x+y-4 t) \\
& -4 f(x-y+t) \\
& -4 f(x+y-3 t) \\
& -24 f(x-t)+6 f(-y+2 t), \\
& F(x+t,-y+2 t)=f(2 x-y+4 t)+f(2 x+y) \\
& -4 f(x-y+3 t) \\
& -4 f(x+y-t) \\
& -24 f(x+t)+6 f(-y+2 t), \\
& F(x, y+4 t)=f(2 x+y+4 t) \\
& +f(2 x-y-4 t) \\
& -4 f(x+y+4 t) \\
& -4 f(x-y-4 t)-24 f(x) \\
& +6 f(y+4 t)
\end{aligned}
$$




$$
\begin{aligned}
& F(x,-y+4 t)=f(2 x-y+4 t) \\
& +f(2 x+y-4 t) \\
& -4 f(x-y+4 t) \\
& -4 f(x+y-4 t)-24 f(x) \\
& +6 f(-y+4 t) \text {, } \\
& F(-t, x+y+2 t)=f(x+y)+f(-x-y-4 t) \\
& -4 f(x+y+t) \\
& -4 f(-x-y-3 t) \\
& -24 f(-t)+6 f(x+y+2 t), \\
& F(t, x-y-2 t)=f(x-y)+f(-x+y+4 t) \\
& -4 f(x-y-t) \\
& -4 f(-x+y+3 t)-24 f(t) \\
& +6 f(x-y-2 t) \text {, } \\
& F(-t, x-y+2 t)=f(x-y)+f(-x+y-4 t) \\
& -4 f(x-y+t) \\
& -4 f(-x+y-3 t) \\
& -24 f(-t)+6 f(x-y+2 t), \\
& F(t, x+y-2 t)=f(x+y)+f(-x-y+4 t) \\
& -4 f(x+y-t) \\
& -4 f(-x-y+3 t)-24 f(t) \\
& +6 f(x+y-2 t) \text {, } \\
& F(-t, x+y+t)=f(x+y-t)+f(-x-y-3 t) \\
& -4 f(x+y) \\
& -4 f(-x-y-2 t) \\
& -24 f(-t)+6 f(x+y+t), \\
& F(t, x-y-t)=f(x-y+t)+f(-x+y+3 t) \\
& -4 f(x-y) \\
& -4 f(-x+y+2 t)-24 f(t) \\
& +6 f(x-y-t) \text {, } \\
& F(-t, x-y+t)=f(x-y-t)+f(-x+y-3 t) \\
& -4 f(x-y) \\
& -4 f(-x+y-2 t) \\
& -24 f(-t)+6 f(x-y+t), \\
& F(t, x+y-t)=f(x+y+t)+f(-x-y+3 t) \\
& -4 f(x+y) \\
& -4 f(-x-y+2 t)-24 f(t) \\
& +6 f(x+y-t) \text {, } \\
& F(t, x+y)=f(x+y+2 t)+f(-x-y+2 t) \\
& -4 f(x+y+t) \\
& -4 f(-x-y+t)-24 f(t) \\
& +6 f(x+y), \\
& F(-t, x-y)=f(x-y-2 t)+f(-x+y-2 t) \\
& -4 f(x-y-t) \\
& -4 f(-x+y-t)-24 f(-t) \\
& +6 f(x-y) \text {, } \\
& F(t, x-y)=f(x-y+2 t)+f(-x+y+2 t) \\
& -4 f(x-y+t) \\
& -4 f(-x+y+t)-24 f(t) \\
& +6 f(x-y), \\
& F(-t, x+y)=f(x+y-2 t)+f(-x-y-2 t) \\
& -4 f(x+y-t) \\
& -4 f(-x-y-t)-24 f(-t) \\
& +6 f(x+y) \text {, } \\
& F(x+t, y)=f(2 x+y+2 t) \\
& +f(2 x-y+2 t) \\
& -4 f(x+y+t) \\
& -4 f(x-y+t) \\
& -24 f(x+t)+6 f(y), \\
& F(x-t, y)=f(2 x+y-2 t) \\
& +f(2 x-y-2 t) \\
& -4 f(x+y-t) \\
& -4 f(x-y-t) \\
& -24 f(x-t)+6 f(y), \\
& F(x, y+2 t)=f(2 x+y+2 t) \\
& +f(2 x-y-2 t) \\
& -4 f(x+y+2 t)
\end{aligned}
$$




$$
\begin{aligned}
& -4 f(x-y-2 t)-24 f(x) \quad F(0, x+y-2 t)=3 f(x+y-2 t) \\
& +6 f(y+2 t) \\
& -3 f(-x-y+2 t) \\
& F(x,-y+2 t)=f(2 x-y+2 t) \\
& +f(2 x+y-2 t) \\
& -4 f(x-y+2 t) \\
& -4 f(x+y-2 t)-24 f(x) \\
& +6 f(-y+2 t) \\
& -24 f(0) \text {, } \\
& F(0, x-y+2 t)=3 f(x-y+2 t) \\
& -3 f(-x+y-2 t) \\
& -24 f(0) \text {, } \\
& F(0, x-y-2 t)=3 f(x-y-2 t) \\
& F(0, x+y+4 t)=3 f(x+y+4 t) \\
& -3 f(-x+y+2 t) \\
& -3 f(-x-y-4 t) \\
& -24 f(0), \\
& -24 f(0) \text {, } \\
& F(0, x+y-4 t)=3 f(x+y-4 t) \\
& -3 f(-x-y+4 t) \\
& -24 f(0) \\
& F(0, x-y+4 t)=3 f(x-y+4 t) \\
& -3 f(-x+y-4 t) \\
& -24 f(0) \\
& F(0, x-y-4 t)=3 f(x-y-4 t) \\
& -3 f(-x+y+4 t) \\
& -24 f(0) \text {, } \\
& F(0, x+y+3 t)=3 f(x+y+3 t) \\
& -3 f(-x-y-3 t) \\
& -24 f(0) \text {, }
\end{aligned}
$$$$
F(0, x+y-3 t)=3 f(x+y-3 t)
$$$$
-3 f(-x-y+3 t)
$$$$
-24 f(0) \text {, }
$$$$
F(0, x-y+3 t)=3 f(x-y+3 t)
$$$$
-3 f(-x+y-3 t)
$$$$
-24 f(0) \text {, }
$$$$
F(0, x-y-3 t)=3 f(x-y-3 t)
$$$$
-3 f(-x+y+3 t)
$$$$
-24 f(0) \text {, }
$$$$
F(0, x+y+2 t)=3 f(x+y+2 t)
$$$$
-3 f(-x-y-2 t)
$$$$
-24 f(0) \text {, }
$$

Thus, we get the functional identity

$$
\begin{aligned}
3 F(x & -t, y+2 t)+3 F(x+t, y+2 t) \\
& +3 F(x-t,-y+2 t)+3 F(x+t,-y+2 t) \\
& -3 F(x, y+4 t)-3 F(x,-y+4 t) \\
& -12 F(-t, x+y+2 t)-12 F(t, x-y-2 t) \\
& -12 F(-t, x-y+2 t)-12 F(t, x+y-2 t) \\
& -36 F(-t, x+y+t)-36 F(t, x-y-t) \\
& -36 F(-t, x-y+t)-36 F(t, x+y-t) \\
& -24 F(t, x+y)-24 F(-t, x-y) \\
& -24 F(t, x-y)-24 F(-t, x+y) \\
& -6 F(x+t, y)-6 F(x-t, y)+6 F(x, y+2 t)
\end{aligned}
$$




$$
\begin{aligned}
& +6 F(x,-y+2 t)-4 F(0, x+y+4 t) \\
& -4 F(0, x+y-4 t)-4 F(0, x-y+4 t) \\
& -4 F(0, x-y-4 t)+4 F(0, x+y+3 t) \\
& +4 F(0, x+y-3 t)+4 F(0, x-y+3 t) \\
& +4 F(0, x-y-3 t)+40 F(0, x+y+2 t) \\
& +40 F(0, x+y-2 t)+40 F(0, x-y+2 t) \\
& +40 F(0, x-y-2 t)+32 F(0, x+y+t) \\
& +32 F(0, x+y-t)+32 F(0, x-y+t) \\
& +32 F(0, x-y-t)+18 F(2 t, y)+216 F(t, 0) \\
& +1152 F(0, t) \\
& =6 f(2 x+y)+6 f(2 x-y)-24 f(x+y) \\
& -24 f(x-y)-144 f(x)+36 f(y) \\
& -33264 f(0)
\end{aligned}
$$

Since $\Omega$ satisfies condition (C), for given $x, y \in X$, there exists $t \in X$ such that

$$
\|F(u, v)\| \leq \epsilon
$$

for all $(u, v) \in P_{x, y, t}$. Thus, dividing (14) by 6 and using the triangle inequality and (15) we have

$$
\begin{aligned}
& \| f(2 x+y)+f(2 x-y)-4 f(x+y)-4 f(x-y) \\
& -24 f(x)+6 f(y)-5544 f(0) \| \leq \frac{1018}{3} \epsilon
\end{aligned}
$$

for all $x, y \in X$. Let $g(x)=f(x)+231 f(0)$ for all $x \in X$. Then from (16) we have

$$
\begin{aligned}
& \| g(2 x+y)+g(2 x-y)-4 g(x+y)-4 g(x-y) \\
& \quad-24 g(x)+6 g(y) \| \leq \frac{1018}{3} \epsilon
\end{aligned}
$$

for all $x, y \in X$. Using Theorem 1 with (17), we get (10). This completes the proof.

Remark 5. Letting $x=0$ in (10) and dividing the result by 232 we have $\|f(0)\| \leq 509 \epsilon /(232 \times 36)$. Thus, inequality (10) implies

$$
\|f(x)-T(x)\| \leq 231\|f(0)\|+\frac{509}{36} \epsilon \leq \frac{235667}{8352} \epsilon
$$

for all $x \in X$.

Let $d \geq 0$. It is easy to see that $\{(x, y) \in X \times X:\|x\|+\|y\| \geq$ d) satisfies condition (C). Indeed, for given $x, y \in X$ if we choose $t \geq\|x\|+\|y\|+d$, then $P_{x, y, t} \subset\{(x, y) \in X \times X$ : $\|x\|+\|y\| \geq d\}$. Thus, as a direct consequence of Theorem 4 we obtain the following result (see $[14,16,17]$ for similar results).
Corollary 6. Let $\epsilon, d \geq 0$ be fixed. Suppose that $f: X \rightarrow Y$ satisfies inequality (2) for all $x, y \in X$ such that $\|x\|+\|y\| \geq$ $d$. Then there exists a unique quartic mapping $T: X \rightarrow Y$ satisfying (10) for all $x \in X$.

In particular, if $\epsilon=0$, we have the following.

Corollary 7. Suppose that $f: X \rightarrow Y$ (1) for all $(x, y) \in \Omega$. Then, (1) holds for all $x, y \in X$.

\section{Stability Problem in a Set of Lebesgue Measure Zero}

In this section, we show that even a set $\Gamma$ of Lebesgue measure zero can satisfy condition (C) when $X=\mathbb{R}$. From now on, we identify $\mathbb{R}^{2}$ with $\mathbb{C}$.

Lemma 8. Let $P=\left\{\left(p_{j}+a_{j} t, q_{j}+b_{j} t\right): j=1,2, \ldots, r\right\}$, where $p_{j}, q_{j}, a_{j}, b_{j} \in \mathbb{R}$ with $a_{j}^{2}+b_{j}^{2} \neq 0$ for all $j=1,2, \ldots, r$. Then there exists $\theta \in[0,2 \pi]$ such that $e^{i \theta} P:=\left\{\left(p_{j}^{\prime}+a_{j}^{\prime} t, q_{j}^{\prime}+b_{j}^{\prime} t\right)\right.$ : $j=1,2, \ldots, r\}$ satisfies $a_{j}^{\prime} b_{j}^{\prime} \neq 0$ for all $j=1,2, \ldots, r$.

Proof. The coefficients $a_{j}^{\prime}$ and $b_{j}^{\prime}$ are given by

$$
\begin{aligned}
& a_{j}^{\prime}=a_{j} \cos \theta-b_{j} \sin \theta, \\
& b_{j}^{\prime}=a_{j} \sin \theta-b_{j} \cos \theta
\end{aligned}
$$

for all $j=1,2, \ldots, r$. Now, the equation

$$
\prod_{j=1}^{r}\left(a_{j} \cos \theta-b_{j} \sin \theta\right)\left(a_{j} \sin \theta-b_{j} \cos \theta\right)=0
$$

has only a finite number of zeros in $[0,2 \pi]$. Thus, we can choose $\theta \in[0,2 \pi]$ such that $\prod_{j=1}^{r} a_{j}^{\prime} b_{j}^{\prime} \neq 0$. This completes the proof.

Lemma 9. One can find a set $K \subset \mathbb{R}$ of Lebesgue measure 0 such that, for any countable subsets $U \subset \mathbb{R}, V \subset \mathbb{R} \backslash\{0\}$, and $M>0$, there exists $\lambda \geq M$ satisfying

$$
U+\lambda V=\{u+\lambda v: u \in U, v \in V\} \subset K
$$

Proof. It is shown in [22, Theorem 1.6] that there exists a set $K \subset \mathbb{R}$ of Lebesgue measure 0 such that $\mathbb{R} \backslash K$ is of the first Baire category; that is, $R \backslash K$ is a countable union of nowhere dense subsets of $\mathbb{R}$. Let $U=\left\{u_{1}, u_{2}, u_{3}, \ldots\right\}$ and $V=\left\{v_{1}, v_{2}, v_{3}, \ldots\right\}$ and $K_{m, n}^{c}=v_{m}^{-1}\left(K^{c}-u_{n}\right), m, n=1,2,3, \ldots$. Then, since $K^{c}$ is of the first Baire category, $K_{m, n}^{c}$ are also of the first Baire category for all $m, n=1,2,3, \ldots$. Since each $K_{m, n}^{c}$ consists of a countable union of nowhere dense subsets, by the Baire category theorem, countably many of them cannot cover $[M, \infty)$; that is,

$$
\bigcup_{m, n=1}^{\infty} K_{m, n}^{c} \nsupseteq[M, \infty) \text {. }
$$


Thus, there exists $\lambda \geq M$ such that $\lambda \notin K_{m, n}^{c}$ for all $m, n=1,2$, $3, \ldots$. This means that $u_{n}+v_{m} \lambda \in K$ for all $m, n=1,2,3, \ldots$. This completes the proof.

Theorem 10. Let $d \geq 0$ be fixed. Then there exists a set $\Gamma_{d} \subset$ $\{(x, y):|x|+|y| \geq d\}$ of 2-dimensional Lebesgue measure 0 which satisfies condition (C).

Proof. Let $P_{x, y, t}$ be the set in condition (C). Then by Lemma 8 we can choose $\theta \in[0,2 \pi]$ such that $e^{i \theta} P_{x, y, t}:=\left\{\left(p_{j}^{\prime}+a_{j}^{\prime} t, q_{j}^{\prime}+\right.\right.$ $\left.\left.b_{j}^{\prime} t\right): j=1,2, \ldots, r\right\}$ satisfies $a_{j}^{\prime} b_{j}^{\prime} \neq 0$ for all $j=1,2, \ldots, r$. Let $K$ be the set in Lemma 9. Then $\Gamma_{d}=e^{-i \theta}(K \times K) \cap\{(x, y)$ : $|x|+|y| \geq d\}$ has 2-dimensional Lebesgue measure 0 . Now, we show that $\Gamma$ satisfies condition $(\mathrm{C})$; that is, for given $x, y \in \mathbb{R}$, there exists $t \in \mathbb{R}$ satisfying the conditions

$$
\begin{aligned}
e^{i \theta} P_{x, y, t} & \subset K \times K, \\
& P_{x, y, t} \subset\{(x, y):|x|+|y| \geq d\} .
\end{aligned}
$$

Let $e^{i \theta} P_{x, y, t}:=\left\{\left(p_{j}^{\prime}+a_{j}^{\prime} t, q_{j}^{\prime}+b_{j}^{\prime} t\right): j=1,2, \ldots, r\right\}, U=$ $\left\{p_{j}^{\prime}, q_{j}^{\prime}: j=1,2, \ldots, r\right\}$, and $V=\left\{a_{j}^{\prime}, b_{j}^{\prime}: j=1,2, \ldots\right\}$. Then we have

$$
\left\{u, v:(u, v) \in e^{i \theta} P_{x, y, t}\right\} \subset U+t V .
$$

Now, by Lemma 9, for given $x, y \in \mathbb{R}$ there exists $t \geq|x|+$ $|y|+d$ such that

$$
U+t V \subset K
$$

From (24) and (25) we have

$$
e^{i \theta} P_{x, y, t} \subset K \times K
$$

Since $t \geq|x|+|y|+d$, using the triangle inequality we have $|u|+|v| \geq d$ for all $(u, v) \in P_{x, y, t}$. Thus, $\Gamma$ satisfies $(\mathrm{C})$. This completes the proof.

Now, as a direct consequence of Theorems 4 and 10 we have the following.

Corollary 11. Let $\epsilon, d \geq 0$ be fixed. Suppose that $f: \mathbb{R} \rightarrow Y$ satisfies inequality (2) for all $(x, y) \in \Gamma_{d}$. Then there exists a unique quartic mapping $T: X \rightarrow Y$ satisfying (10) for all $x \in$ $\mathbb{R}$.

As a consequence of Corollary 11 we obtain an asymptotic behavior of $f$ satisfying condition (11) as $|x|+|y| \rightarrow \infty$ only for $(x, y) \in \Gamma_{0}$.

Corollary 12. Suppose that $f: \mathbb{R} \rightarrow Y$ satisfies condition (11). Then $f$ is a quartic mapping.

Proof. Condition (11) implies that, for each $n \in \mathbb{N}$, there exists $d_{n}>0$ such that

$$
\begin{aligned}
& \| f(2 x+y)+f(2 x-y)-4 f(x+y)-4 f(x-y) \\
& -24 f(x)+6 f(y) \| \leq \frac{1}{n}
\end{aligned}
$$

for all $(x, y) \in \Gamma_{d_{n}}$. By Corollary 11, there exists a unique quartic mapping $T_{n}: X \rightarrow Y$ such that

$$
\left\|f(x)-T_{n}(x)+231 f(0)\right\| \leq \frac{509}{36 n}
$$

for all $x \in X$. Replacing $n$ by $m$ in (28) and using the triangle inequality we have

$$
\left\|T_{m}(x)-T_{n}(x)\right\| \leq \frac{509}{36 n}+\frac{509}{36 m} \leq \frac{509}{18}
$$

for all $x \in X$. Let $T_{m, n}(x)=T_{m}(x)-T_{n}(x)$ for all $x \in X$. Then by (29), $T_{m, n}$ is a bounded quartic mapping. Thus, we have $T_{m, n}=0$ and hence $T_{m}=T_{n}:=T$ for all $m, n \in \mathbb{N}$. Letting $n \rightarrow \infty$ in (28) we have $f(0)=0$ and hence $f(x)=T(x)$ for all $x \in X$. This completes the proof.

Remark 13. If we define $\Gamma \subset \mathbb{R}^{2 n}$ as an appropriate rotation of $2 n$-product $K^{2 n}$ of $K$, then $\Gamma_{d}$ has $2 n$-dimensional measure 0 and satisfies condition (C). Consequently, we obtain the following.

Theorem 14. Suppose that $f: \mathbb{R}^{n} \rightarrow Y$ satisfies inequality (2) for all $(x, y) \in \Gamma_{d}$. Then there exists a unique quartic mapping $T: \mathbb{R}^{n} \rightarrow Y$ satisfying (10) for all $x \in \mathbb{R}^{n}$.

\section{Competing Interests}

The authors declare that there are no competing interests regarding the publication of this paper.

\section{Acknowledgments}

This research was supported by the Basic Science Research Program through the National Research Foundation of Korea (NRF) funded by the Ministry of Education (no. 2015R1D1A3A01019573).

\section{References}

[1] S. H. Lee, S. M. Im, and I. S. Hwang, "Quartic functional equations," Journal of Mathematical Analysis and Applications, vol. 307, no. 2, pp. 387-394, 2005.

[2] A. Bodaghi, "Stability of a quartic functional equation," The Scientific World Journal, vol. 2014, Article ID 752146, 9 pages, 2014.

[3] D. Kang, "On the stability of generalized quartic mappings in quasi- $\beta$-normed spaces," Journal of Inequalities and Applications, vol. 2010, Article ID 198098, 11 pages, 2010.

[4] C. Alsina and J.-L. Garcia-Roig, "On a conditional Cauchy equation on rhombuses," in Functional Analysis, Approximation theory and Numerical Analysis, J. M. Rassias, Ed., pp. 5-7, World Scientific, River Edge, NJ, USA, 1994.

[5] B. Batko, "Stability of an alternative functional equation," Journal of Mathematical Analysis and Applications, vol. 339, no. 1, pp. 303-311, 2008.

[6] B. Batko, "On approximation of approximate solutions of Dhombres' equation," Journal of Mathematical Analysis and Applications, vol. 340, no. 1, pp. 424-432, 2008. 
[7] J. Brzdęk, "On the quotient stability of a family of functional equations," Nonlinear Analysis: Theory, Methods \& Applications, vol. 71, no. 10, pp. 4396-4404, 2009.

[8] J. Brzdęk, "On a method of proving the Hyers-Ulam stability of functional equations on restricted domains," The Australian Journal of Mathematical Analycis and Applications, vol. 6, no. 1, pp. 1-10, 2009.

[9] A. Bahyrycz and J. Brzdęk, "On solutions of the d'Alembert equation on a restricted domain," Aequationes Mathematicae, vol. 85, no. 1-2, pp. 169-183, 2013.

[10] J. Brzdęk and J. Sikorska, "A conditional exponential functional equation and its stability," Nonlinear Analysis: Theory, Methods \& Applications, vol. 72, no. 6, pp. 2923-2934, 2010.

[11] J.-Y. Chung, "Stability of functional equations on restricted domains in a group and their asymptotic behaviors," Computers and Mathematics with Applications, vol. 60, no. 9, pp. 26532665, 2010.

[12] R. Ger and J. Sikorska, "On the Cauchy equation on spheres," Annales Mathematicae Silesianae, vol. 11, pp. 89-99, 1997.

[13] S.-M. Jung, Hyers-Ulam-Rassias Stability of Functional Equations in Nonlinear Analisis, vol. 48, Springer, New York, NY, USA, 2011.

[14] S.-M. Jung, "On the Hyers-Ulam stability of the functional equations that have the quadratic property," Journal of Mathematical Analysis and Applications, vol. 222, no. 1, pp. 126-137, 1998.

[15] M. Kuczma, "Functional equations on restricted domains," Aequationes Mathematicae, vol. 18, no. 1-2, pp. 1-34, 1978.

[16] J. M. Rassias and M. J. Rassias, "On the Ulam stability of Jensen and Jensen type mappings on restricted domains," Journal of Mathematical Analysis and Applications, vol. 281, no. 2, pp. 516524, 2003.

[17] J. M. Rassias, "On the Ulam stability of mixed type mappings on restricted domains," Journal of Mathematical Analysis and Applications, vol. 276, no. 2, pp. 747-762, 2002.

[18] J. Sikorska, "On two conditional Pexider functinal equations and their stabilities," Nonlinear Analysis. Theory, Methods \& Applications A: Theory and Methods, vol. 70, no. 7, pp. 26732684, 2009.

[19] F. Skof, "Sull'approssimazione delle applicazioni localmente $\delta$ dadditive," Atti dell'Accademia delle Scienze di Torino, vol. 117, pp. 377-389, 1983.

[20] J. Chung and J. M. Rassias, "On a measure zero stability problem of a cyclic equation," Bulletin of the Australian Mathematical Society, vol. 93, no. 2, pp. 272-282, 2016.

[21] J. Chung, Y. M. Ju, and J. M. Rassias, "Cubic functional equation on restricted domains of Lebesgue measure zero," Canadian Mathematical Bulletin, In press.

[22] J. C. Oxtoby, Measure and Category, vol. 2, Springer, New York, NY, USA, 2nd edition, 1980. 


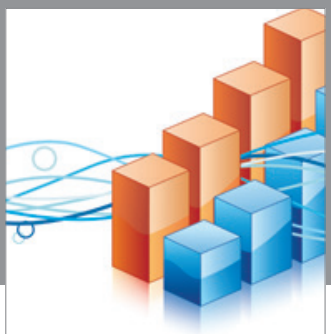

Advances in

Operations Research

vatem alat4

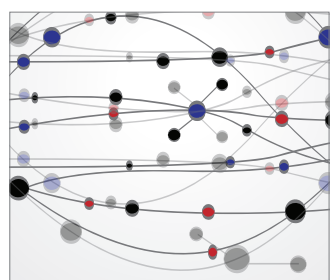

\section{The Scientific} World Journal
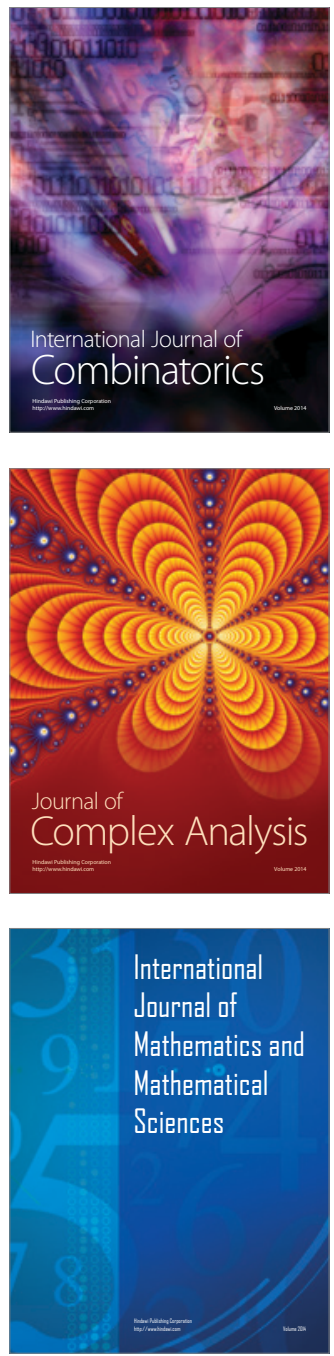
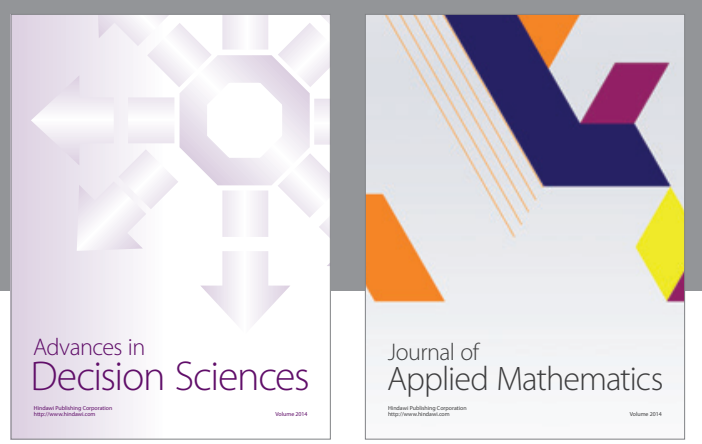

Algebra

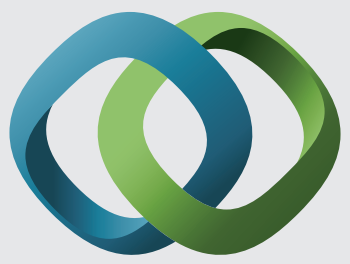

\section{Hindawi}

Submit your manuscripts at

http://www.hindawi.com
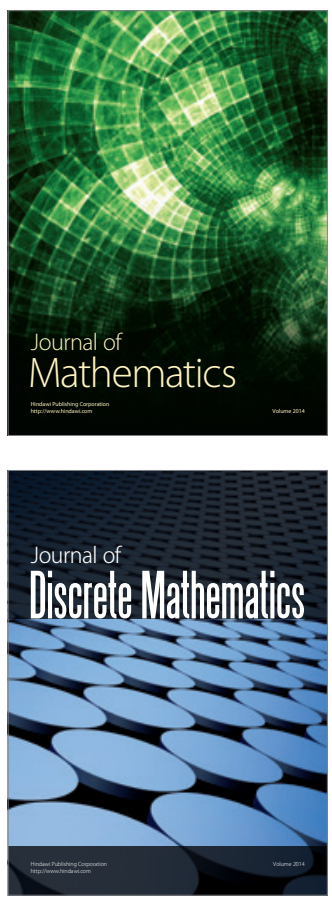

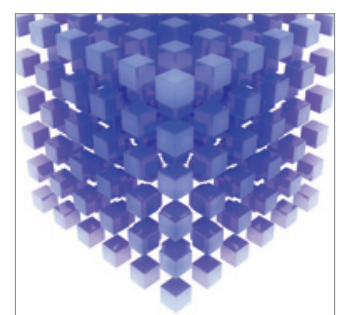

Mathematical Problems in Engineering
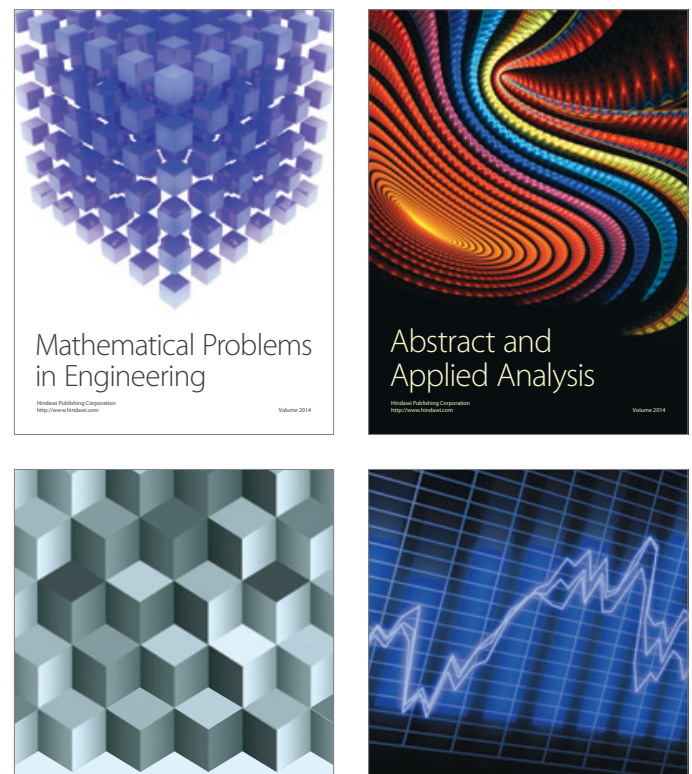

Journal of

Function Spaces

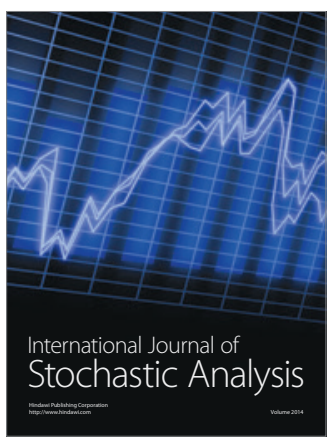

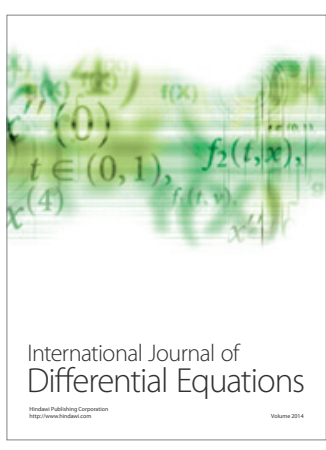
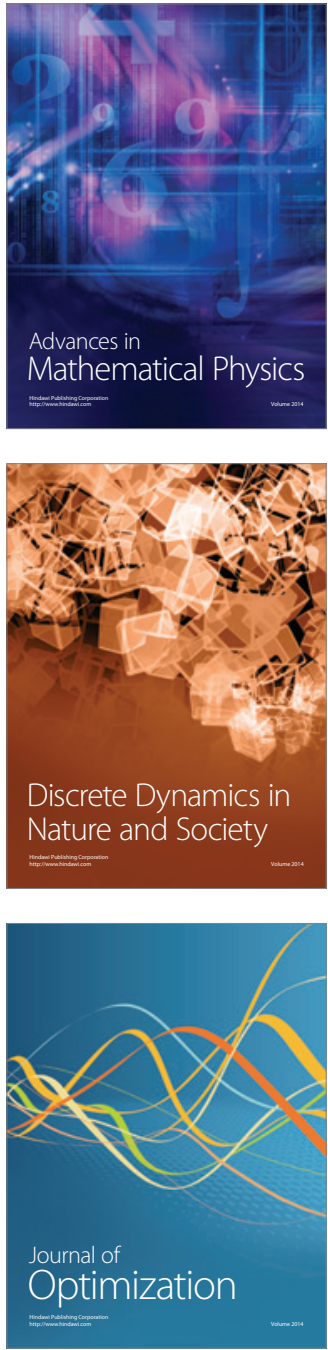